\title{
Plebański formulation of general relativity: a practical introduction
}

\author{
Kirill Krasnov
}

Received: 23 November 2009 / Accepted: 12 July 2010 / Published online: 31 July 2010

(C) The Author(s) 2010. This article is published with open access at Springerlink.com

\begin{abstract}
We give a pedagogical introduction into an old, but unfortunately not commonly known formulation of GR in terms of self-dual two-forms due to in particular Jerzy Plebański. Our presentation is rather explicit in that we show how the familiar textbook solutions: Schwarzschild, Volkoff-Oppenheimer, as well as those describing the Newtonian limit, a gravitational wave and the homogeneous isotropic Universe can be obtained within this formalism. Our description shows how Plebański formulation gives quite an economical alternative to the usual metric and frame-based schemes for deriving Einstein equations.
\end{abstract}

Keyword Self-dual formulation of general relativity

\section{Plebanski formulation of general relativity}

The aim of this short paper is to give a description of Plebański self-dual formulation [1] of general relativity (GR) in a version that we found most suited for practical computations. Our presentation is very explicit, in that the standard textbook solutions of GR are obtained. As we shall see, given an ansatz for the metric, Plebański formulation produces Einstein equations even more quickly than the already efficient tetrad method. In our opinion, the efficiency and beauty of this formulation may warrant its inclusion in general relativity textbooks.

Our convention for the signature is $(-,+,+,+)$. We start with a collection of historical remarks.

K. Krasnov $(\varangle)$

School of Mathematical Sciences, University of Nottingham,

Nottingham, NG7 2RD, UK

e-mail: kirill.krasnov@nottingham.ac.uk 


\subsection{Historical remarks}

The basic objects of Plebański's formulation of GR are self-dual two-forms. These objects have appeared in the GR literature much before Plebański paper [1]. In fact, the famous Petrov's classification [2] of "spaces defining gravitational fields" already uses self-dual (and anti-self-dual) bivectors in a key way. Thus, the theorem proved by Petrov states that the gravitational field (solution of vacuum Einstein equations) can be classified according to algebraic types of a complex symmetric $3 \times 3$ matrix obtained as a complex linear combination of the diagonal and off-diagonal blocks of the Riemann tensor viewed as a symmetric tensor in the linear space of bivectors. Self-dual (and anti-self-dual) bivectors then naturally appear as principal bivectors of the Riemann tensor. A completely analogous but more modern treatment that forms the $3 \times 3$ matrix in question as the complex linear combination of the "electric" and "magnetic" parts of the Weyl tensor was given in [3]. Again, the self- and anti-self-dual bivectors are central in these considerations.

It was then remarked in [4] that the $3 \times 3$ complex matrix encoding the Weyl curvature can be computed directly, i.e. avoiding computing the Riemann curvature first. This can be done by elementary operations of differentiation if one forms the self-dual complex linear combinations from the components of the Levi-Civita tetrad-compatible connection. This encodes 24 real components of the Levi-Civita connection in 12 complex components of a self-dual connection. The $3 \times 3$ matrix of Weyl curvature components is then computed as the curvature of this self-dual connection.

The self-dual two-forms first appeared in a pioneering paper [5]. This paper uses the null tetrad formalism and thus also provides a link to the spinor formalism of Penrose [6]. Thus, the spinor formalism combines the 24 real rotation coefficients into 12 complex Newmann-Penrose spin coefficients [7], which is similar to what happens in the self-dual formalism. The paper [5] for the first time writes equations for the self-dual connection one-forms as those in terms of exterior derivatives of the self-dual twoforms. It also clearly states that the isomorphism between the Lorentz group $\operatorname{SO}(1,3)$ and the complexified rotation group $\mathrm{SO}(3, \mathbb{C})$ is what is at the root of the self-dual formalism. Finally, the Einstein equations are very clearly stated in this paper as the condition that the curvature of the self-dual connection is self-dual. Another exposition of the formalism for GR based on differential forms and self-duality is that of [8].

Yet another presentation of the self-dual formalism for GR appeared in [9]. This reference is very close in spirit to the exposition of the present paper. One important new point in this reference is the emphasis it places on the role played by the Hodge duality operator, which is interpreted as defining the complex structure in the space of two-forms. Similar to [5], (vacuum) Einstein equations are stated here as the condition that the curvature of the self-dual connection is self-dual.

The self-dual formalism for general relativity was taken further by Plebański in [1]. The paper cited uses spinor notations, but it can be easily translated into somewhat more easily readable SO(3) notations used in e.g. [9]. The main novelty of Plebański's work is that for the first time the main object of the theory is taken to be not a metric from whose tetrads the self-dual two-forms are constructed, but rather a triple of two-forms satisfying certain additional equations. These equations guarantee that the two-forms in question are obtained from tetrads, and thus provide a link to the usual 
metric formulation. The paper [1] also gave a remarkably simple action principle realizing these ideas. The basic dynamical field in this action is a triple of two-forms and no metric ever appears. Later Ashtekar's new Hamiltonian formulation of general relativity [10] was found [11] to be just the phase space version of Plebański's theory.

Our presentation does not use the action principle [1], and so all the technology employed here is already available in earlier works [5,9]. Thus, it might perhaps be objectionable to name the self-dual formulation of GR presented here after Plebański, since many earlier works contain the same ideas. However, the self-dual formulation of general relativity has become firmly associated with the name of Plebański in the quantum gravity community that uses the action principle of [1] as the starting point for quantization of gravity, so this terminology is standard at least in some part of the GR community. Moreover, in our opinion it does take a considerable courage to propose a formulation of gravity in which the theory is not about a metric. We believe that this justifies attributing the self-dual two-form formulation to Jerzy Plebański.

We proceed with a description of the key idea in the usual tensor notations.

\subsection{Einstein condition and the Hodge operator}

Given a spacetime metric $g_{\mu \nu}$ the condition that this metric is Einstein reads: $R_{\mu \nu} \sim$ $g_{\mu \nu}$, where $R_{\mu \nu}:=R_{\mu}{ }^{\rho}{ }_{\nu}$ is the Ricci tensor, and the fact that the proportionality coefficient in this condition must be a constant is implied by the (differential) Bianchi identity $\nabla^{\mu} G_{\mu \nu}=0$, where $\nabla_{\mu}$ is the metric-compatible derivative operator and $G_{\mu \nu}=R_{\mu \nu}-(1 / 2) g_{\mu \nu} R$ is the Einstein tensor. As usual, the quantity $R$ is the Ricci scalar $R:=R_{\mu}^{\mu}$, and all indices are raised and lowered with the metric. The self-dual formulation of GR is based on the following simple and well-known reformulation of the Einstein condition (vacuum Einstein equations) in terms of the Hodge operator. Thus, let us introduce the operation of Hodge dual that acts on bivectors (anti-symmetric rank two tensors) $A_{\mu \nu}$ :

$$
A_{\mu \nu} \rightarrow{ }^{*} A_{\mu \nu}=\frac{1}{2} \epsilon_{\mu \nu}^{\rho \sigma} A_{\rho \sigma}
$$

where the quantity $\epsilon_{\mu \nu \rho \sigma}$ is the volume 4-form for the metric $g_{\mu \nu}$. The following elementary properties of the Hodge operator are easily verified: its square is minus one and it is invariant under conformal transformations of the metric $g \rightarrow \Omega^{2} g$.

Given the Riemann curvature tensor $R_{\mu \nu \rho \sigma}$ one can apply the Hodge operator to either the first or the second pair of indices:

$$
{ }^{*} R_{\mu \nu \rho \sigma}:=\frac{1}{2} \epsilon_{\mu \nu}{ }^{\mu^{\prime} v^{\prime}} R_{\mu^{\prime} \nu^{\prime} \rho \sigma}, \quad R_{\mu \nu \rho \sigma}^{*}:=\frac{1}{2} R_{\mu \nu \rho^{\prime} \sigma^{\prime}} \epsilon_{\rho \sigma}^{\rho^{\prime} \sigma^{\prime}} .
$$

It is a straightforward computation to check that the Einstein condition $R_{\mu \nu} \sim g_{\mu \nu}$ (together with the first Bianchi identity $R_{\mu[v \rho \sigma]}=0$ ) is equivalent to the condition that the left and right Hodge duals of the Riemann tensor coincide:

$$
R_{\mu \nu} \sim g_{\mu \nu} \Longleftrightarrow{ }^{*} R_{\mu \nu \rho \sigma}=R_{\mu \nu \rho \sigma}^{*} .
$$


Indeed, since $(*)^{2}=-1$ the last condition is equivalent to $R_{\mu \nu \rho \sigma}=-{ }^{*} R_{\mu \nu \rho \sigma}^{*}$. Contracting a pair of indices on the left-hand-side to produce the Ricci curvature, and expanding the product of two epsilons on the right-hand-side in terms of products of the metric tensor, one finds $R_{\mu \nu}=(1 / 4) g_{\mu \nu} R$, which is the Einstein condition.

To arrive at the self-dual formulation we need a slight additional reformulation of the condition (3). Thus, let us use the Hodge operator (1) to introduce projectors on the spaces of the so-called self- and anti-self-dual bivectors. These are bivectors that remain essentially unchanged under the Hodge duality:

$$
{ }^{*} A^{\mu \nu}=i A^{\mu \nu} \Longleftrightarrow \text { (self-dual), }{ }^{*} A^{\mu \nu}=-i A^{\mu \nu} \Longleftrightarrow \text { (anti-self-dual). }
$$

The corresponding projectors are:

$$
P_{\mu \nu}^{ \pm \rho \sigma}=\frac{1}{2}\left(I_{\mu \nu}{ }^{\rho \sigma} \pm \frac{1}{2 i} \epsilon_{\mu \nu}{ }^{\rho \sigma}\right),
$$

where we have introduced the identity operator in the space of bivectors:

$$
I_{\mu \nu}^{\rho \sigma}:=\delta_{\mu}^{[\rho} \delta_{v}^{\sigma]}=\frac{1}{2}\left(\delta_{\mu}^{\rho} \delta_{v}^{\sigma}-\delta_{\mu}^{\sigma} \delta_{\nu}^{\rho}\right)
$$

It is then easy to show that the condition (3) can be rewritten using the above projectors as follows:

$$
\text { (Ricci) } \sim \text { (metric) } \Longleftrightarrow P^{-} R P^{+}=0,
$$

where the anti-self-dual projector is applied on the left and the self-dual projector is applied on the right. Another convenient way to state this is to say that the Einstein condition is equivalent to the statement that the self-dual part of the Riemann curvature with respect to the second pair of indices is self-dual with respect to the first pair as well. As we have already described in the previous subsection, this reformulation of the Einstein condition has been known for a long time, see e.g. [5,9]. We also note that in the mathematics literature, see e.g. Chapter 13 of [12], this is known as the Atiyah-Hitchin-Singer theorem [13]. A proof of equivalence of (7) to (3) is an elementary exercise using the definition of the projector operators. We can now use the observation (7) as the basis of a new formulation of general relativity.

\subsection{Plebański self-dual formulation: pure gravity}

Let us present the self-dual formulation as a concrete recipe for obtaining Einstein equations starting from a metric. We will then explain why this recipe is equivalent to (7). Our description is quite analogous to that in [9]. Thus, this reference already emphasizes the key fact that in the self-dual method the number of equations to be solved is halved as compared to the tetrad method.

The first step of this formulation is to find a tetrad for the spacetime metric. Thus, one represents the metric as: $d s^{2}=\theta^{I} \otimes \theta^{J} \eta_{I J}$, where $\theta^{I}$ are the tetrad one-forms, 
$I=0,1,2,3$ are "internal" indices, and $\eta_{I J}$ is the Minkowski metric. The next step is to construct two-forms $\theta^{I} \wedge \theta^{J}$ and then take their self-dual parts with respect to the indices $I J$. More concretely, one introduces an arbitrary time plus space split of the internal indices $I=(0, i), i=1,2,3$, and considers the following triple of two-forms:

$$
\Sigma^{i}:=i \theta^{0} \wedge \theta^{i}-\frac{1}{2} \epsilon^{i j k} \theta^{j} \wedge \theta^{k}
$$

As is not hard to check, the two-forms $\Sigma^{i}$ satisfy:

$$
\begin{gathered}
\frac{i}{2} \Sigma^{i} \wedge \Sigma^{j}=\delta^{i j} \sqrt{-g} d^{4} x, \\
\Sigma^{i} \wedge \bar{\Sigma}^{j}=0,
\end{gathered}
$$

where the anti-self-dual forms $\bar{\Sigma}^{i}$ are given by:

$$
\bar{\Sigma}^{i}:=i \theta^{0} \wedge \theta^{i}+\frac{1}{2} \epsilon^{i j k} \theta^{j} \wedge \theta^{k}
$$

The next step, after the two-forms $\Sigma^{i}$ are written down, is to find a connection $A^{i}$ that is "compatible" with the triple $\Sigma^{i}$, i.e. the connection that satisfies:

$$
d \Sigma^{i}+\epsilon^{i j k} A^{j} \wedge \Sigma^{k}=0
$$

This is an algebraic equation for the components of the connection $A^{i}$ that has a unique solution. It is not hard to write down an explicit expression for $A^{i}$ in terms of the derivatives $d \Sigma^{i}$, but in practice it is easier to solve the Eq. (12) for each problem at hand by expanding it in the coordinate three-forms. We shall denote the $\Sigma$-compatible connection by $A_{\Sigma}$. It is not hard to verify that $A_{\Sigma}$ is just the self-dual part of the tetrad-compatible $d \theta^{I}+\omega_{J}^{I} \wedge \theta^{J}=0$ connection $\omega^{I J}$, i.e., $A^{i}=i \omega^{0 i}-(1 / 2) \epsilon^{i j k} \omega^{j k}$. This fact will be important below for our explanation of the self-dual method from the point of view of (7), but it is not essential if one just want to follow the method.

The final step is to compute the curvature

$$
F^{i}=d A^{i}+\frac{1}{2} \epsilon^{i j k} A^{j} \wedge A^{k}
$$

of the connection $A_{\Sigma}$. It is not hard to show that this is just the self-dual part of the curvature $F^{I J}(\omega)$ of the tetrad-compatible spin-connection $\omega^{I J}$, but, once again, this is not essential if one just wants to follow the method. Curvature is a two-form, and can be split in the basis of self-dual $\Sigma^{i}$ and anti-self-dual $\bar{\Sigma}^{i}$ two-forms. Thus, one can always write:

$$
F^{i}\left(A_{\Sigma}\right)=F^{i j} \Sigma^{j}+\bar{F}^{i j} \bar{\Sigma}^{j}
$$


The matrices $F^{i j}, \bar{F}^{i j}$ are the main quantities of interest for the Einstein equations can be written quite easily as the following ten conditions:

$$
\operatorname{Tr}(F)=-\Lambda, \quad \bar{F}^{i j}=0,
$$

where $\Lambda$ is the cosmological constant. As a bonus of this method, one not only obtains Einstein equations, but also automatically gets an expression for the part of the curvature not constrained by Einstein equations - the Weyl curvature. Thus, the self-dual part $\Psi^{i j}$ of the Weyl curvature tensor is given by the tracefree part of the matrix $F^{i j}$ : $\Psi^{i j}=\left(F^{i j}\right)_{t f}$.

The described method of obtaining Einstein equations is quite efficient for practical computations. The steps one has to take to arrive at (15) are similar to the steps one makes in the tetrad formulation. The advantage of working with two-forms $\Sigma^{i}$ instead of tetrads $\theta^{I}$ is that one has half equations to deal with at intermediate steps, at the expense of all quantities becoming complex. We shall see the power of this method below when we use it to obtain the standard solutions of GR.

An explanation of this method from first principles, i.e. from Eq. (7) is as follows. As we have already said, the $\Sigma$-compatible connection $A^{i}$ turns out to be just the self-dual part of the tetrad-compatible spin connection $\omega^{I J}$. This means that the curvature $F^{i}\left(A_{\Sigma}\right)$ is just the self-dual part of the curvature tensor $R^{I J}(\omega)$. Now the second equation in (15) just says that the curvature $F^{i}\left(A_{\Sigma}\right)$ is self-dual as a twoform. However, because of the noted relation between $F^{i}$ and $R^{I J}$ this is equivalent to the condition that the self-dual part of $R^{I J}$ is self-dual as a two-form, which is just the condition (7). The first equation in (15) can be shown to be a consequence of the Bianchi identity $D_{A} F^{i}=0$, analogous to how the proportionality coefficient in the Einstein condition $R_{\mu \nu} \sim g_{\mu \nu}$ is shown to be a constant related to the cosmological constant. For more discussion on abstract aspects of Plebański self-dual formulation of GR the reader may consult [5,9] and also [14].

\subsection{Coupling to matter}

In the previous subsection we have described the vacuum theory. We now need to extend it to the case when a non-zero stress-energy tensor is present. This is not hard to do in full generality, but does not appear to have been spelled out in the literature. Only the case of Einstein-Maxwell theory is commented upon in [5].

To describe the coupling to matter it is necessary to split the stress-energy tensor of matter $T_{\mu \nu}$ into its trace $T=T_{\mu}^{\mu}$ and the tracefree $\tilde{T}_{\mu \nu}=T_{\mu \nu}-(1 / 4) g_{\mu \nu} T$ parts, and then form out of the tracefree part a $3 \times 3$ (complex) matrix

$$
T^{i j}=\tilde{T}_{\mu}^{\rho} \Sigma_{v \rho}^{i} \bar{\Sigma}^{j \mu \nu}
$$

where, as before $\Sigma^{i}, \bar{\Sigma}^{i}$ are the self- and anti-self-dual forms (8) and (11). The nonvacuum Einstein Eq. (15) then take the following simple form:

$$
\operatorname{Tr}(F)=-\Lambda-2 \pi G T, \quad \bar{F}^{i j}=-2 \pi G T^{i j} .
$$


For the ideal fluid, which is what matters for most practical applications, we have $T_{\mu \nu}=(\rho+P) u_{\mu} u_{v}+P g_{\mu \nu}$, where $\rho, P$ are the energy and pressure densities correspondingly, and $u_{\mu}$ is the 4-velocity vector. The trace of the stress-energy tensor is given by $T=(3 P-\rho)$, the tracefree part $\tilde{T}_{\mu \nu}=(\rho+P)\left(u_{\mu} u_{\nu}+(1 / 4) g_{\mu \nu}\right)$ and so we get:

$$
T^{i j}=(\rho+P)\left(\frac{\delta^{i j}}{1-|u|^{2}}-2 i \epsilon^{i j k} \frac{u^{k}}{\sqrt{1-|u|^{2}}}\right) \text {, }
$$

where $u^{k}$ is just the spatial component of the normalized $u_{\mu} u^{\mu}=-1$ velocity 4-vector:

$$
u_{\mu}=\frac{1}{\sqrt{1-|u|^{2}}}\left((d t)_{\mu}+u^{i}\left(d x^{i}\right)_{\mu}\right)
$$

In these two formulas $|u|^{2}=u^{i} u^{i}$, with, as usual, a sum over the repeated index $i$ implied. These are Minkowski spacetime expressions. A generalization to other spacetime metrics is straightforward. Other types of matter can be described similarly.

We are now ready to study some simple solutions of GR using the formulation described. We start with the graviton solution of the linearized theory. We shall set the cosmological constant $\Lambda$ to zero from now on.

\section{Gravitational wave solution}

The gravitational wave is a solution of linearized around Minkowski spacetime vacuum field equations. The background two-forms are given by:

$$
\Sigma_{0}^{i}=i d t \wedge d x^{i}-\frac{1}{2} \epsilon^{i j k} d x^{j} \wedge d x^{k}
$$

In Plebanski formulation the graviton is described as a perturbation:

$$
\Sigma^{i}=\Sigma_{0}^{i}+\delta \Sigma^{i}
$$

The perturbation two-forms $\delta \Sigma^{i}$ can then be decomposed into the background twoforms:

$$
\delta \Sigma^{i}=b^{i j} \Sigma_{0}^{j}+\bar{b}^{i j} \bar{\Sigma}_{0}^{j}
$$

In terms of the matrices $b^{i j}, \bar{b}^{i j}$ the reality conditions (9), (10) become

$$
b^{i j}=b \delta^{i j}+\omega^{i j}, \quad \bar{b}^{i j}=\left(\bar{b}^{i j}\right)^{*},
$$

where $b$ is a real scalar and $\omega^{i j}$ is an arbitrary (complex) anti-symmetric matrix. The second equation implies that the matrix of quantities $\bar{b}^{i j}$ is real. 
The representation (22) of a general perturbation immediately provides a convenient classification of perturbations. Indeed, the matrix $\bar{b}^{i j}$ can be split into its symmetric traceless, anti-symmetric, and the trace parts, while the matrix $\omega^{i j}$ is already antisymmetric. Then the transverse part of an symmetric tracefree matrix describes a tensor mode, while the transverse part of an anti-symmetric matrix describes a vector one. For example, perturbations of the tensor type that correspond to gravitational waves are given by:

$$
\delta_{\text {tens }} \Sigma^{i}=h^{i j} \bar{\Sigma}^{j}
$$

where $h^{i j}$ is a symmetric, traceless, transverse $h_{, i}^{i j}=0$ three by three matrix.

Perturbations of the scalar and vector types can also be considered but the modes they describe are non-propagating. Further, some of these modes are gauge. For example, the modes described by $\omega^{i j}$ part of the perturbation are just the gauge modes corresponding to availability of (complexified) $\mathrm{SO}(3)$ transformations in this framework. Other modes are gauge for the infinitesimal diffeomorphisms, which in this framework are described by:

$$
\delta_{\xi} \Sigma^{i}=\mathcal{L}_{\xi} \Sigma^{i}=d \iota_{\xi} \Sigma^{i}
$$

where $\mathcal{L}_{\xi}$ is the Lie derivative along an infinitesimal vector field $\xi$ and $\iota \xi$ is the operation of the interior product a vector field with a form. It is quite easy to compute (25) and see which modes can be set to zero by the diffeomorphisms. One finds that, for example, the gauge-fixed perturbation of the scalar type is described by:

$$
\delta_{\text {scal }} \Sigma^{i}=\phi \Sigma^{i}+\psi \bar{\Sigma}^{i}
$$

where $\phi, \psi$ are related to the usual Newtonian potentials $\Phi, \Psi$ in an elementary way. We shall further consider scalar perturbations below when we study the Newtonian limit.

Having determined the form of the perturbation that we would like to study, it is elementary to obtain the linearized Einstein equations. Thus, the first step is to find the linearized connection $\delta A^{i}$ such that: $d \delta \Sigma^{i}+\epsilon^{i j k} \delta A^{j} \wedge \Sigma^{k}=0$. This is a simple exercise in algebra with the result being:

$$
\delta A^{i}=\left(-h_{, l}^{i k} \epsilon^{j k l}+i\left(h^{i j}\right)^{\prime}\right) d x^{j}
$$

Here the prime denotes the time derivative. The corresponding linearized curvature is also easy to compute. We decompose the answer into the self- and anti-self-dual background forms:

$$
d \delta A^{i}=\frac{1}{2}\left(h^{i j}\right)^{\prime \prime}\left(\Sigma^{j}+\bar{\Sigma}^{j}\right)+\frac{1}{2} \Delta h^{i j}\left(\Sigma^{j}-\bar{\Sigma}^{j}\right)+i\left(h_{, l}^{i k}\right)^{\prime} \epsilon^{j k l} \Sigma^{j} .
$$

Now setting to zero the anti-self-dual terms on the right hand-side gives the Einstein equations: 


$$
\left(h^{i j}\right)^{\prime \prime}-\Delta h^{i j}=\square h^{i j}=0,
$$

whose solutions are plane waves. As a bonus, we also get an expression for the matrix of the components of the self-dual part of the Weyl curvature of the linearized solution describing the gravitational wave:

$$
\Psi^{i j}=\frac{1}{2}\left(\left(h^{i j}\right)^{\prime \prime}+\Delta h^{i j}+2 i\left(h_{, l}^{i k}\right)^{\prime} \epsilon^{j k l}\right)
$$

which, as is easy to check, is symmetric and traceless.

\section{Schwarzschild solution}

In this case one is still interested in vacuum field equations. We start from the usual expression for a spherically symmetric metric:

$$
d s^{2}=-f^{2}(r) d t^{2}+g^{2}(r) d r^{2}+r^{2} d \Omega^{2},
$$

where $d \Omega^{2}$ is the usual metric on the unit sphere. A tetrad one-form is given by:

$$
e^{t}=f(r) d t, \quad e^{r}=g(r) d r, \quad e^{\theta}=r d \theta, \quad e^{\phi}=r \sin (\theta) d \phi .
$$

A convenient set of self-dual combinations (8) is then:

$$
\Sigma^{1}=i e^{t} \wedge e^{r}-e^{\theta} \wedge e^{\phi}, \quad \Sigma^{2}=i e^{t} \wedge e^{\theta}-e^{\phi} \wedge e^{r}, \quad \Sigma^{3}=i e^{t} \wedge e^{\phi}-e^{r} \wedge e^{\theta} .
$$

We now have to find the associated $\mathfrak{s u}(2)$ connection $A^{i}$. One decomposes the connection into coordinate one-forms, and then solves the arising system of linear equations for the coefficients. This gives:

$$
A^{1}=\frac{i f^{\prime}}{g} d t+\cos (\theta) d \phi, \quad A^{2}=-\frac{\sin (\theta) d \phi}{g}, \quad A^{3}=\frac{d \theta}{g},
$$

where, as usual, prime denotes the derivative with respect to the $r$ coordinate. It is not hard to verify that the above connection solves (12).

In practice finding the connection is the most time consuming task. After this is done, it only remains to compute the curvature. This is a simple exercise in differentiation. One gets:

$$
\begin{aligned}
& F^{1}=d A^{1}+A^{2} \wedge A^{3}=-\left(\frac{i f^{\prime}}{g}\right)^{\prime} d t \wedge d r-\left(1-\frac{1}{g^{2}}\right) \sin (\theta) d \theta \wedge d \phi, \\
& F^{2}=d A^{2}+A^{3} \wedge A^{1}=-\frac{g^{\prime}}{g^{2}} \sin (\theta) d \phi \wedge d r-\frac{i f^{\prime}}{g^{2}} d t \wedge d \theta, \\
& F^{3}=d A^{3}+A^{1} \wedge A^{2}=-\frac{g^{\prime}}{g^{2}} d r \wedge d \theta-\frac{i f^{\prime}}{g^{2}} \sin (\theta) d t \wedge d \phi .
\end{aligned}
$$


In the final step of extracting Einstein equations one simply has to express the coordinate two-forms appearing in (35) in terms of the self- and anti-self-dual forms $\bar{\Sigma}^{i}=-\left(\Sigma^{i}\right)^{*}$. Thus, our final expression for the curvature components is:

$$
\begin{aligned}
F^{1} & =-\frac{1}{2 f g}\left(\frac{f^{\prime}}{g}\right)^{\prime}\left(\bar{\Sigma}^{1}+\Sigma^{1}\right)-\frac{1}{2 r^{2}}\left(1-\frac{1}{g^{2}}\right)\left(\bar{\Sigma}^{1}-\Sigma^{1}\right), \\
F^{2} & =-\frac{1}{2 g^{2} r}\left(\frac{g^{\prime}}{g}\left(\bar{\Sigma}^{2}-\Sigma^{2}\right)+\frac{f^{\prime}}{f}\left(\bar{\Sigma}^{2}+\Sigma^{2}\right)\right), \\
F^{3} & =-\frac{1}{2 g^{2} r}\left(\frac{g^{\prime}}{g}\left(\bar{\Sigma}^{3}-\Sigma^{3}\right)+\frac{f^{\prime}}{f}\left(\bar{\Sigma}^{3}+\Sigma^{3}\right)\right) .
\end{aligned}
$$

After one has an expression for the curvature in terms of the basis two-forms $\Sigma^{i}, \bar{\Sigma}^{i}$ one can immediately read off Einstein equations. First, one should equate to zero all the anti-self-dual components of the curvature. This immediately gives (from $F^{2}, F^{3}$ ):

$$
\frac{f^{\prime}}{f}+\frac{g^{\prime}}{g}=0,
$$

whose solution is the familiar $f=1 / g$. The equation coming from the anti-self-dual part of $F^{3}$ reads:

$$
\frac{1}{f g}\left(\frac{f^{\prime}}{g}\right)^{\prime}+\frac{1}{r^{2}}\left(1-\frac{1}{g^{2}}\right)=0 .
$$

The other equation, namely the condition that the trace part of the self-dual part of the curvature is zero reads, with (37), (38) taken into account, after some simple rewriting:

$$
\left(\frac{1}{g^{2}}\right)^{\prime}=\frac{1}{r}\left(1-\frac{1}{g^{2}}\right),
$$

which immediately gives the famous:

$$
\frac{1}{g^{2}}=1-\frac{r_{+}}{r}
$$

which also solves (38), as is not hard to see. The sign of the integration constant here is chosen so that the spacetime obtained is that of positive total gravitational mass. The integration constant itself is chosen so that $r=r_{+}$is the place where $g^{-2}(r)$ vanishes.

We would like to emphasize how much more thought-economizing the above derivation was as compared to the usual (e.g. tetrad-based) derivation given in all the textbooks. Even the steps leading to the computation of the curvature involve just the half of operations needed in the tetrad scheme (in the above spherically-symmetric case the economy is probably not as good, as a large number of coefficients is anyway zero). However, what really is most economical about Plebanski formulation is that one can immediately read off the equations from the expression for the curvature, 
unlike in the usual tetrad-based scheme. Indeed, in the tetrad scheme one first has to form the Ricci tensor, and only after that write Einstein equations. In this last step one has to raise and lower indices, which introduces some tricky minus signs, making it a non-trivial exercise to get the right final expressions. In the case of Plebański formulation this last step is absent altogether, the manipulations leading to Einstein equations are as algorithmic as all the previous ones, making it much harder to make a mistake.

\section{Volkoff-Oppenheimer solution}

In this section we switch on the stress-energy of matter. We have computed the curvature components in the previous section. The equations that one obtains from the anti-self-dual components of the curvature are:

$$
\begin{gathered}
\frac{1}{2 g^{2} r}\left(\frac{g^{\prime}}{g}+\frac{f^{\prime}}{f}\right)=2 \pi G(\rho+P), \\
\frac{1}{2 f g}\left(\frac{f^{\prime}}{g}\right)^{\prime}+\frac{1}{2 r^{2}}\left(1-\frac{1}{g^{2}}\right)=2 \pi G(\rho+P) .
\end{gathered}
$$

The equation one obtains from the trace of the self-dual part is given by:

$$
-\frac{1}{g^{2} r}\left(\frac{f^{\prime}}{f}-\frac{g^{\prime}}{g}\right)-\frac{1}{2 f g}\left(\frac{f^{\prime}}{g}\right)^{\prime}+\frac{1}{2 r^{2}}\left(1-\frac{1}{g^{2}}\right)=2 \pi G(\rho-3 P) .
$$

Let us now take twice the Eq. (41) summed with (42), and subtract the result from the Eq. (43). We get, after some simple rewriting:

$$
\frac{1}{r}\left(1-\frac{1}{g^{2}}\right)^{\prime}+\frac{1}{r^{2}}\left(1-\frac{1}{g^{2}}\right)=8 \pi G \rho
$$

which is the non-zero $\rho$ generalization of the Eq. (39). It immediately gives:

$$
g^{-2}(r)=1-\frac{2 m}{r}, \quad m(r)=\int_{0}^{r} 4 \pi G \rho r^{2}
$$

The function $f$ can then be found from e.g. Eq. (41). Substituting (45) we get:

$$
\frac{f^{\prime}}{f}=\frac{m+4 \pi G P r^{3}}{r(r-2 m)}
$$

integrating which we get $f$. This ODE should be solved with the "boundary" condition $f^{2}(R)=1-2 M / R$, where $M, R$ are the total mass and radius of the spherical object in question. 


\section{Newtonian limit}

Let us now consider the case of small (static) perturbations around the Minkowski spacetime background (20) described by two scalar potentials $\phi, \psi$ :

$$
\delta B^{i}=\phi \Sigma^{i}+\psi \bar{\Sigma}^{i}
$$

Here we assume the functions $\phi, \psi$ to be only those of the spatial coordinates (time independent). It can be easily shown that all other scalar perturbations can be set to zero by a diffeomorphism and an $\mathrm{SO}(3)$ rotation. It is easy to check that (47) corresponds to the following perturbation of the metric:

$$
d s^{2}=-(1+2 \Phi) d t^{2}+(1-2 \Psi) \sum_{i} d x_{i}^{2}
$$

with

$$
\Phi=\frac{\phi+3 \psi}{2}, \quad \Psi=\frac{\psi-\phi}{2}
$$

We now have to solve the linearized "compatibility" equation and obtain the linearized connection. We can solve for the $\phi$ and $\psi$ parts of the perturbation separately. We get:

$$
A_{\phi}^{i}=\frac{i}{2} \phi_{, i} d t-\frac{1}{2} \epsilon^{i j k} \phi_{, k} d x^{j}, \quad A_{\psi}^{i}=\frac{3 i}{2} \psi_{, i} d t+\frac{1}{2} \epsilon^{i j k} \psi_{, k} d x^{j}
$$

The corresponding linearized curvatures are:

$$
\begin{aligned}
& d A_{\phi}^{i}=-\frac{1}{4} \Delta \phi \Sigma^{i}+\frac{1}{4}\left(-2 \phi_{, i j}+\delta^{i j} \Delta \phi\right) \bar{\Sigma}^{j} \\
& d A_{\psi}^{i}=-\frac{1}{4}\left(4 \psi_{, i j}-\delta^{i j} \Delta \psi\right) \Sigma^{j}-\frac{1}{4}\left(2 \psi_{, i j}+\delta^{i j} \Delta \psi\right) \bar{\Sigma}^{j}
\end{aligned}
$$

Let us now analyze the Plebanski Eq. (17) with zero pressure $P=0$ and velocity $u^{i}=0$. First, the anti-self-dual part on the right-hand-side is diagonal, which requires of the off-diagonal components to vanish:

$$
\left((\phi+\psi)_{, i j}\right)_{t f}=0,
$$

where $t f$ denotes the trace-free part. This implies $\phi+\psi=0$, or $\Psi=\Phi$. Both of the diagonal components then give the Laplace equation, which in terms of the potentials (49) takes the familiar form:

$$
\Delta \Phi=4 \pi G \rho .
$$


We also get for free an expression for the components of the Weyl tensor:

$$
\Psi^{i j}=\Phi^{, i j}-\frac{1}{3} \delta^{i j} \Delta \Phi
$$

which, we note, is real, as is typical of static backgrounds.

\section{Homogeneous isotropic Universe}

Using Plebanski formalism it is also quite easy to obtain the Friedman equations describing the evolution of a homogeneous isotropic Universe. The corresponding metric is given by:

$$
d s^{2}=a^{2}(\eta)\left(-d \eta^{2}+\sum_{i}\left(d x^{i}\right)^{2}\right)
$$

where $\eta$ is the conformal time. The corresponding self- and anti-self-dual two-forms are given by:

$$
\begin{aligned}
& \Sigma^{i}=a^{2}\left(i d \eta \wedge d x^{i}-\frac{1}{2} \epsilon^{i j k} d x^{j} \wedge d x^{k}\right), \\
& \bar{\Sigma}^{i}=a^{2}\left(i d \eta \wedge d x^{i}+\frac{1}{2} \epsilon^{i j k} d x^{j} \wedge d x^{k}\right) .
\end{aligned}
$$

The connection $A^{i}$ compatible with the set of self-dual two-forms $\Sigma^{i}$, i.e. satisfying (12), is given by:

$$
A^{i}=i \mathcal{H} d x^{i}
$$

where we have introduced:

$$
\mathcal{H}:=\frac{a^{\prime}}{a}
$$

and the prime denotes the derivative with respect to the conformal time. The curvature $F^{i}=d A^{i}+(1 / 2) \epsilon^{i j k} A^{j} \wedge A^{k}$ of the connection (57) is given by:

$$
F^{i}=\frac{1}{2 a^{2}}\left(\mathcal{H}^{\prime}+\mathcal{H}^{2}\right) \Sigma^{i}+\frac{1}{2 a^{2}}\left(\mathcal{H}^{\prime}-\mathcal{H}^{2}\right) \bar{\Sigma}^{i}
$$

From (59), (17) we immediately get Einstein equations:

$$
\frac{1}{2 a^{2}}\left(\mathcal{H}^{\prime}+\mathcal{H}^{2}\right)=\frac{2 \pi G}{3}(\rho-3 P), \quad \frac{1}{2 a^{2}}\left(\mathcal{H}^{\prime}-\mathcal{H}^{2}\right)=-2 \pi G(\rho+P) .
$$


These are more customarily known as their linear combinations:

$$
\mathcal{H}^{2}=\frac{8 \pi G a^{2} \rho}{3}, \quad 2 \mathcal{H}^{\prime}+\mathcal{H}^{2}=-8 \pi G a^{2} p .
$$

\section{Discussion}

We would like to conclude this short paper by emphasizing once more how computationally efficient the Plebański self-dual formulation of general relativity is as compared to other standard textbook methods such as that based on tetrads. For this reason its inclusion in GR textbooks may be warranted, also in view of its conceptual simplicity and beauty.

What we have not discussed in this paper is if Plebański formulation can be not just a tool for obtaining Einstein equations but also for solving them. To this end we just note that the Bianchi identity takes in this formulation a very simple form:

$$
D_{A} \Psi^{i j} \wedge \Sigma^{j}=0
$$

where $\Psi^{i j}$ is the matrix of self-dual components of the Weyl curvature tensor. In the case of the Schwarzschild solution the matrix $\Psi^{i j}$ is diagonal $\Psi^{i j}=$ $\beta(r) \operatorname{diag}(2,-1,-1)$, where $\beta(r)$ is a function of the radial coordinate. The Bianchi identity (62) then becomes a first order differential equation for $\beta$, which immediately gives $\beta \sim 1 / r^{3}$. One then finds that one of the Einstein equations is an algebraic relation between $g(r)$ and $\beta(r)$, so the problem of solving the system of Einstein equations reduces in this case to that of solving (62). This simple observation is relevant in situations other than Schwarzschild, and in some cases serves as an efficient tool for obtaining solutions.

As we have already mentioned in the "historical remarks" subsection, the described here method is closely related to that of Newman-Penrose [15], with the later being, loosely speaking, the two-component spinor version of the self-dual two-form one. In addition to working with self-dual quantities, and expressing all quantities in terms of spinors, the Newman-Penrose formalism introduces and works with a doubly-null tetrad, which is quite powerful, but at the same time makes the Lorentz-covariance of the theory not manifest. In contrast, Lorentz rotations of a tetrad in Plebański formalism are described very simply as (complexified) $\mathrm{SO}(3)$ rotations. It is also considerably more easy to work with spacetime forms than with spinors. For these reasons Plebański method may be an convenient middle ground between the relatively cumbersome tetrad method and a very powerful, but somewhat too relying on working in components Newman-Penrose formalism.

Acknowledgements The author was supported by an EPSRC Advanced Fellowship. The author is grateful to an anonymous referee for suggesting a list of references that grew into the "historical remarks" subsection.

Open Access This article is distributed under the terms of the Creative Commons Attribution Noncommercial License which permits any noncommercial use, distribution, and reproduction in any medium, provided the original author(s) and source are credited. 


\section{References}

1. Plebanski, J.F.: On the separation of Einsteinian substructures. J. Math. Phys. 18, 2511 (1977)

2. Petrov, A.Z.: The classification of spaces defining gravitational fields (in Russian). In: Scientific Proceedings of Kazan State University named after V. I. Ulyanov-Lenin, vol. 114, pp. 55-69 (1954); English translation published in Gen. Relativ. Gravit. vol. 32, pp. 1661 (2000)

3. Jordan, P., Ehlers, J., Kundt, W.: Strenge Lösungen der Feldgleichungen der Allgemeinen Relativitätstheorie, Akademie der Wissenschaften and der Literatur, Abhandlungen der Mathematisch-naturwissenschaftliche Klasse, Nr 2, pp. 21-105 (1960); English translation published in Gen. Relativ. Gravit., vol. 41, pp. 2191-2280 (2009)

4. Taubes, A.H.: The Riemann-Christoffel tensor and tetrad and self-dual formalisms. In: Hoffmann, B. (ed.) Perspectives in Geometry (Essays in honor of V. Hlavaty), pp. 360-368. Indiana University Press, Bloomington (1966)

5. Cahen, M., Debever, R., Defrise, L.: A complex vectorial formalism in general relativity. J. Math. Mech. 16, 761-785 (1967)

6. Penrose, R.: A spinor approach to general relativity. Ann. Phys. 10, 171 (1960)

7. Newman, E., Penrose, R.: An approach to gravitational radiation by a method of spin coefficients. J. Math. Phys. 3, 566 (1962)

8. Israel, W.: Differential forms in general relativity. Comm. Dublin Inst. Adv. Studies, Ser. A 19 (1970)

9. Brans, C.H.: Complex structures and representations of the Einstein equations. J. Math. Phys. 15, 1559 (1974)

10. Ashtekar, A.: New Hamiltonian formulation of general relativity. Phys. Rev. D 36, 1587 (1987)

11. Jacobson, T., Smolin, L.: Covariant action for Ashtekar's form of canonical gravity. Class. Quant. Gravit. 5, 583 (1988)

12. Besse, Arthur. I.: Einstein Manifolds. Springer, Berlin (1987)

13. Atiyah, M.F., Hitchin, N.J., Singer, I.M.: Selfduality in four-dimensional Riemannian geometry. Proc. R. Soc. Lond. A 362, 425 (1978)

14. Capovilla, R., Jacobson, T., Dell, J., Mason, L.: Selfdual two forms and gravity. Class. Quant. Gravit. 8, 41 (1991)

15. Penrose, R. Rindler, W.: Spinors and Space-Time. 1. Two Spinor Calculus and Relativistic Fields. Cambridge Monographs On Mathematical Physics, vol. 458, Cambridge: Univ. Pr. (1984) 